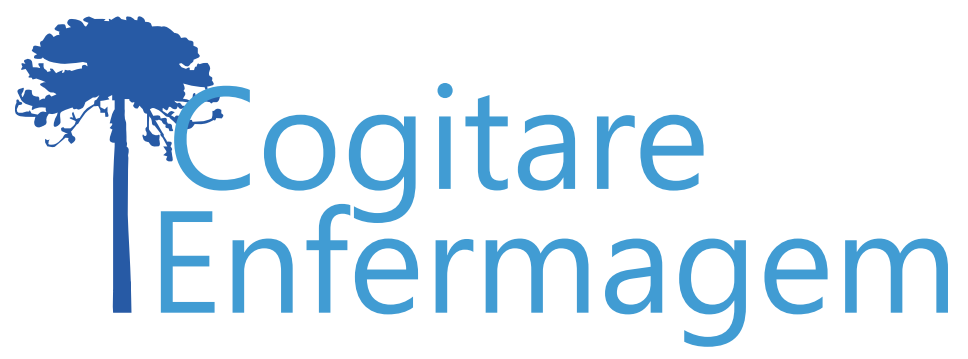

\title{
QUALIDADE DE VIDA DE USUÁRIOS COM DOENÇAS CRÔNICAS NÃO TRANSMISSÍVEIS ASSISTIDOS NA ATENÇÃO PRIMÁRIA À SAÚDE
}

\section{QUALITY OF LIFE OF USERS WITH CHRONIC NON-COMMUNICABLE DISEASES ASSISTED IN PRIMARY HEALTH CARE}

\author{
Pâmela Naíse Pasquetti ${ }^{1}$ (i) \\ Adriane Cristina Bernat Kolankiewicz ${ }^{2}$ (1) \\ Gabriela Ceretta Flôres ${ }^{2}$ (1) \\ Vanessa Dalsasso Batista Winter ${ }^{2}$ (i) \\ Leticia Flores Trindade ${ }^{2}$ (1) \\ Laura Renner Bandeira² ${ }^{2}$ (i) \\ Marli Maria Loro ${ }^{2}$ (1)
}

\begin{abstract}
Objective: to evaluate the Quality of Life and its association with demographic and clinical characteristics of users with Chronic Noncommunicable Diseases, assisted in Primary Health Care. Method: cross-sectional study with individuals diagnosed with chronic non-communicable disease, developed with sociodemographic/clinical questionnaire and WHOQOL-Bref, in Primary Health Care in a municipality in the northwest of Rio Grande do Sul - Brazil, between July 2018 and June 2019. Results: quality of life with lower mean in the Physical domain (59.71) and higher in the psychological domain (73.30). Results with statistical difference for age group in the Physical $(p<0.048)$, Psychological $(p=0.041)$ and Environment $(p=0.003)$ domains, and with the variables color and profession in the Environment domain $(p=0.002)$. Conclusion: the study contributes to direct and strengthen health education actions developed by primary care teams, aiming at quality of life.
\end{abstract}

DESCRIPTORS: Chronic Disease; Primary Health Care; Quality of Life; Noncommunicable Diseases; Community Health Nursing.

COMO REFERENCIAR ESTE ARTIGO:

Pasquetti PN, Kolankiewicz ACB, Flôres GC, Winter VDB, Trindade LF, Bandeira LR, et al. Qualidade de vida de usuários com doenças crônicas não transmissíveis assistidos na atenção primária à saúde. Cogit. Enferm. [Internet]. 2021 [acesso em "colocar data de acesso, dia, mês abreviado e ano"]; 26. Disponível em: http://dx.doi. org/10.5380/ce.v26i0.75515. 
$\mathrm{Na}$ contemporaneidade, as Doenças Crônicas não Transmissíveis (DCNT) são um problema de saúde pública, em decorrência de seu aumento gradativo nas últimas décadas. Elas representam a maior carga de morbimortalidade no Brasil, o que impulsiona mudanças rápidas e frequentes referente ao aspecto sociodemográfico e clínico dos usuários que buscam atendimento na Atenção Primária a Saúde (APS) ${ }^{(1-2)}$.

Entre as principais causas de óbitos estão as doenças cardiovasculares, respiratórias crônicas, neoplasias e diabetes. Fatores que favorecem o seu desenvolvimento são genéticos, sexo, idade, hábitos e comportamentos de risco como inatividade física, alimentação inadequada, obesidade, tabagismo e uso abusivo de bebidas alcoólicas ${ }^{(1)}$.

Tais condições sobrecarregam os serviços de saúde e influenciam nas demandas de cuidado, uma vez que, segundo o Ministério da Saúde (MS), aproximadamente 57,4 milhões de brasileiros possuem pelo menos uma DCNT, implicando no aumento da procura dos usuários pelos serviços de saúde ${ }^{(2)}$. Nesta perspectiva, cabe aos profissionais da APS identificar os usuários com DCNT em seu território e intervir precocemente, visto o impacto negativo na vida deles. O MS ressalta que a APS deve ser o primeiro contato dos usuários e comunidade com o serviço de saúde pública, e que tem responsabilidade de atender às demandas em saúde dos adscritos a um território definido(3).

O desenvolvimento das DCNT, em sua maioria, tem progressão lenta, com períodos de remissão e exacerbação, sendo, às vezes, inexistente a cura ${ }^{(4)}$. Nessa perspectiva, sabese que indivíduos com DCNT podem ter comprometimento à saúde física e emocional, com potencial de refletir na sua Qualidade de Vida (QV). Para que os usuários com esses agravos mantenham uma QV adequada, é importante avaliar as especificidades de cada sujeito no seu contexto(5), além das formas de enfrentamento e o quanto estas influenciam na rotina do indivíduo, pois possibilitam ampliar as ações em saúde com vistas a melhorar a QV.

O Grupo de Qualidade de Vida da Organização Mundial da Saúde (OMS) conceitua a QV como a percepção do indivíduo sobre sua posição na vida, no contexto do sistema de valores nos quais ele vive, e em relação aos seus objetivos, expectativas e preocupações ${ }^{(6)}$. Autores pontuam que tal conceito abarca aspectos relativos à saúde física, estado psicológico, nível de funcionalidade, sociabilidade e relações com as características ambientais ${ }^{(7)}$. Desta forma, ao reconhecer que indivíduos com DCNT têm sua QV modificada, mensurar estes aspectos faz-se importante, pois pacientes crônicos abrangem uma gama de sintomas físicos, sociais, psicológicos e espirituais relacionados com a progressão natural da doença e os efeitos adversos que a permeiam ${ }^{(8)}$.

Nesse interim, o presente estudo justifica-se pelo crescente número de usuários com diagnóstico de DCNT que acessam diariamente os serviços de saúde com diferentes demandas. Avaliar a QV destes é importante para o planejamento dos cuidados na perspectiva multiprofissional, uma vez que permite tecer e implementar cuidados de forma integral e personalizado ao longo do ciclo vital. Neste contexto, tem-se como objetivo avaliar a QV e a sua relação com as características demográficas e clínicas de usuários com DCNT, assistidos na APS.

\section{MÉTODO}

Estudo transversal, de natureza quantitativa e caráter analítico. Trata-se de um recorte do projeto institucional denominado "Atenção Primária à Saúde, como cenário de 
Cuidado em Saúde e Qualidade de Vida de usuários dos serviços". Foi desenvolvido na APS de um município do noroeste do estado do Rio Grande do Sul (RS).

Os participantes foram selecionados por conveniência, entre julho de 2018 e junho 2019. Para verificar a representatividade estatística, realizou-se cálculo amostral a partir da média de usuários adscritos na APS do referido município (60.000), multiplicando-se este total pelo número de Unidades de Saúde. Partindo-se de um percentual estimado de 0,5\%, um erro amostral de 0,05 e um intervalo de confiança de 95\%, foi necessária a participação mínima de 383 usuários, com vistas a garantir representatividade estatística. Deste total, 216 indivíduos tinham diagnóstico de DCNT.

Foram incluídos indivíduos maiores de 18 anos, com diagnóstico médico de DCNT, assistidos na APS do município, e excluídos aqueles que apresentavam dificuldades em responder os questionários, avaliados pelos bolsistas e voluntários. A coleta de dados foi realizada por bolsistas e voluntárias do projeto, previamente capacitadas; inicialmente, foi solicitado aos acadêmicos/coletores leitura prévia sobre a temática a fim de adquirir embasamento teórico.

Primeiramente, os usuários foram convidados a participar do estudo. Aos concordantes, explanou-se sobre o objetivo, que a participação possuía caráter voluntário e poderia ser interrompida a qualquer tempo. Em seguida, foi apresentado o Termo de Consentimento Livre e Esclarecido. Para garantir a privacidade do participante, após a entrega dos instrumentos, eles foram encaminhados para uma sala reservada na estrutura física da unidade e/ou residência, conforme preferência do entrevistado. Como instrumento de coleta, utilizou-se questionário de caracterização sociodemográfica e clínica, desenvolvido pelos pesquisadores e bolsistas envolvidos no projeto de pesquisa, o qual abordou questões como: faixa etária, sexo, cor/raça, status da profissão, renda familiar, tipo de doença, tempo de diagnóstico e periodicidade das consultas.

Foi utilizado o instrumento WHOQOL-Bref abreviado, versão em português, proposta pela OMS, desenvolvido pelo The WHOQOL Group e validada para o Brasil(9). O instrumento é composto por 26 questões, sendo duas gerais, e as demais representam cada uma das 24 facetas que compõem o instrumento original, divididas em quatro domínios: "físico" (dor física e desconforto, dependência de medicação/tratamento, energia e fadiga, mobilidade, sono e repouso, atividades da vida cotidiana, capacidade para o trabalho), "psicológico" (sentimentos positivos e negativos, espiritualidade/crenças pessoais, aprendizado/memória/concentração, aceitação da imagem corporal e aparência, autoestima), "relações sociais" (relações pessoais, atividade sexual, suporte/apoio social) e "ambiente" (segurança física, ambiente físico, recursos financeiros, novas informações/ habilidades, recreação e lazer, ambiente no lar, cuidados de saúde, transporte) $)^{(9)}$.

As questões são formuladas para respostas em escalas tipo Likert, e incluem intensidade ("nada" a "extremamente"), capacidade ("nada" a "completamente"), frequência ("nunca" a "sempre") e avaliação ("muito insatisfeito" a "muito satisfeito"; "muito ruim" a "muito bom"). As pontuações de cada domínio foram transformadas numa escala de zero a 100 e expressas em termos de médias, onde médias mais altas sugerem melhor percepção de $\mathrm{OV}(9)$.

Os dados foram digitados e tabulados no software SPSS® (Statistic Package for the Social Sciences, versão 25) para Windows ${ }^{\circledR}$. Na estatística descritiva, foram utilizadas distribuições absolutas $(n)$ e relativas (\%), bem como medidas de tendência central e variabilidade, com estudo de normalidade da distribuição de dados pelo teste de Kolmorogov-Smirnov. A comparação dos dados contínuos entre os dois grupos independentes ocorreu pelo teste paramétrico t-Student e, quando a comparação envolveu três ou mais grupos, foi empregada a técnica de Análise de Variância - Post Hoc Sheffé, empregado nas situações em que foi evidenciada a rejeição da hipótese de homogeneidade de variância entre os grupos comparados. Para a decisão sobre os testes de hipóteses serem significativos, adotou-se o nível de significância de $5 \%$. 
A pesquisa foi aprovada pelo Comitê de Ética em Pesquisa da instituição sob parecer n. 2.758.802.

\section{RESULTADOS}

Participaram do estudo 216 usuários. Destes, 68 (31,5\%) encontravam-se na faixa etária entre 61 e 70 anos, com predomínio do sexo feminino 141 (65,3\%). Em relação à cor/raça autorreferida, $160(74,1 \%)$ declararam-se da cor branca. Relacionado ao status profissional, $125(57,9,7 \%)$ eram inativos e $83(38,4 \%)$ aposentados por tempo de trabalho. $O$ maior percentual dos entrevistados possuía renda familiar entre um e dois salários mínimos $155(71,8 \%)$. As doenças mais prevalentes foram as cardiovasculares $68(31,5 \%)$, seguida das neoplasias $40(18,5 \%)$. Quanto ao tempo de doença, $79(36,6 \%)$ tinham diagnóstico há um a três anos. Em relação à periodicidade de consultas, $87(40,3 \%)$ buscavam pelo atendimento mensalmente, conforme a Tabela 1.

Tabela 1 - Perfil Sociodemográfico e clínico de usuários com diagnóstico de Doenças Crônicas não Transmissíveis atendidos na Atenção Primária à Saúde. ljuí, RS, Brasil, 2019 (continua)

\begin{tabular}{lcc} 
Variáveis & N & $\%$ \\
\hline Faixa etária & & \\
\hline 18 a 40 & 23 & 10,7 \\
\hline 41 a 50 & 36 & 16,7 \\
\hline 51 a 60 & 52 & 24,1 \\
\hline 61 a 70 & 68 & 31,5 \\
\hline 71 a 80 & 26 & 12 \\
\hline 81 ou mais & 11 & 5,1 \\
\hline Sexo & & \\
\hline Feminino & 141 & 65,3 \\
\hline Masculino & 75 & 34,7 \\
\hline Cor/raça & & \\
\hline Branca(o) & 160 & 74,1 \\
\hline Parda(o) & 45 & 20,8 \\
\hline Amarela(o) / Negra(o) & 11 & 5,1 \\
\hline Status da profissão & & \\
\hline Ativo & 84 & 38,9 \\
\hline Inativo & 125 & 57,9 \\
\hline Status inativo & & \\
\hline Aposentado por tempo de trabalho & 53 \\
\hline Aposentado por invalidez & 12 \\
\hline Auxílio doença & 38,4 \\
\hline
\end{tabular}




\begin{tabular}{lcc}
\hline Renda familiar & & \\
\hline Menos que um salário & 17 & 7,9 \\
\hline 1 a 2 salários & 155 & 71,8 \\
\hline 3 a 5 salários & 39 & 18,1 \\
\hline 3 a 5 salários & 3 & 1,4 \\
\hline Tipo de doença & & \\
\hline Cardiovasculares & 68 & 31,5 \\
\hline Neoplasias & 40 & 18,5 \\
\hline Diabetes & 38 & 17,6 \\
\hline Doenças Renal Crônica & 29 & 13,4 \\
\hline Tempo de diagnóstico & & \\
\hline Menos de 1 ano & 30 & 13,9 \\
\hline 1 a 3 anos & 79 & 36,6 \\
\hline 3 a 5 anos & 32 & 14,4 \\
\hline 5 anos ou mais & 74 & 34,3 \\
\hline Periodicidade de consultas & & \\
\hline Semanalmente & 12 & 5,6 \\
\hline Quinzenalmente & 18 & 8,3 \\
\hline Mensalmente & 87 & 40,3 \\
\hline Semestralmente & 28,7 \\
\hline Anualmente & 15,3 \\
\hline
\end{tabular}

Fonte: Autores (2019)

Ao avaliar a confiabilidade do instrumento, obteve-se Alfa de Cronbach geral=0,898. A QV com a média mais elevada foi no domínio Psicológico $(73,3 \pm 13,7)$, enquanto a menor média foi detectada no domínio Físico $(59,7 \pm 17,6)$ (Tabela 2).

Tabela 2 - Distribuição dos escores médios da Qualidade de Vida geral e por domínio do WHOQOL-bref. ljuí, RS, Brasil, 2019

\begin{tabular}{lccccccc} 
Domínios & Média & DP & Min & Max & $\mathbf{2 5}^{\circ}$ & $\mathbf{5 0}^{\circ}$ & $\mathbf{7 5}^{\circ}$ \\
\hline Psicológico & 73,3 & 13,7 & 29,1 & 100 & 66,6 & 75 & 79,1 \\
\hline Meio Ambiente & 68,1 & 13,9 & 31,2 & 100 & 59,3 & 65,6 & 56,2 \\
\hline Relações Sociais & 71,7 & 15,1 & 16,6 & 100 & 66,6 & 75 & 83,3 \\
\hline Físico & 59,7 & 17,6 & 10,7 & 100 & 46,2 & 60,7 & 71,4
\end{tabular}

DP = Desvio Padrão

Fonte: Autores (2019) 
Quanto à faixa etária, obteve-se resultados significativos nos domínios Físico $(p<0,048)$, Psicológico $(p=0,041)$ e Meio Ambiente $(p=0,003)$. Referente à variável cor/ raça e status da profissão, houve diferença estatisticamente significativa no domínio Meio Ambiente $(p=0,002)$. Na situação status inativo, foram significativos os domínios Relações Sociais $(p=0,021)$ e Meio Ambiente $(p=0,042)$.

A renda familiar foi a variável que mais impactou a $\mathrm{QV}$, pois diferiu significativamente em todos os domínios: Físico $(p=0,006)$, Psicológico $(p=0,001)$, Relações Sociais $(p=0,000)$, Meio Ambiente $(p=0,001)$ e QV Global $(p=0,005)$. Em relação ao tempo de diagnóstico, houve representatividade nos domínios Relações Sociais $(p=0,042)$ e Meio Ambiente $(p=0,031)$. Sobre a periodicidade das consultas, as diferenças significativas mostraram-se presentes nos domínios Psicológico $(p=0,012)$, Relações Sociais $(p=0,038)$, Meio Ambiente $(p=0,000)$ e QV Global $(p=0,002)$ (Tabela 3).

Tabela 3 - Média e desvio padrão da Qualidade de Vida geral e domínios, conforme características demográficas e clínicas. ljuí, RS, Brasil, 2019 (continua)

Variáveis

Domínios Qualidade de vida WHOQOL-Bref

\begin{tabular}{cccccccccc}
\hline \multicolumn{1}{c}{ Físico } & \multicolumn{2}{c}{ Psicológico } & \multicolumn{2}{c}{$\begin{array}{c}\text { Relações } \\
\text { sociais }\end{array}$} & \multicolumn{2}{c}{$\begin{array}{c}\text { Meio } \\
\text { ambiente }\end{array}$} & QV global \\
\hline Média & DP & Média & DP & Média & DP & Média & DP & Média & DP
\end{tabular}

Faixa etária

\begin{tabular}{lcccccccccc}
\hline De 18 a 40 & 55 & 17,3 & 66,3 & 16,1 & 69,7 & 18,5 & 62 & 13,4 & 69,2 & 12 \\
\hline 41 a 50 & 58,3 & 20 & 70,6 & 17,2 & 70,6 & 15,6 & 63 & 15,4 & 69,4 & 11,7 \\
\hline 51 a 60 & 61,9 & 18,8 & 74,5 & 13,7 & 73,9 & 15,5 & 68,9 & 13,3 & 70,8 & 13,6 \\
\hline 61 a 70 & 61,4 & 16,1 & 74,6 & 11,9 & 71,8 & 13,4 & 69,6 & 13,2 & 74,1 & 13 \\
\hline 71 a 80 & 63 & 13,8 & 78 & 10,5 & 73,4 & 12,5 & 75,4 & 11,6 & 73,1 & 13,5 \\
\hline Acima de 81 & 45,8 & 16,4 & 72 & 8,6 & 65,9 & 20,9 & 69,6 & 13,6 & 69,1 & 17 \\
\hline p-valor & 0,048 & 0,041 & 0,615 & 0,003 & 0,394 \\
\hline
\end{tabular}

Cor/raça

\begin{tabular}{lcccccccccc}
\hline Branca & 60,3 & 18,4 & 74,4 & 13,5 & 72,6 & 15,3 & 70,1 & 14,3 & 72,6 & 12,9 \\
\hline Parda & 57,5 & 14,7 & 69,3 & 14,6 & 68,9 & 14,1 & 63,8 & 11,2 & 68 & 13,9 \\
\hline Amarela, negra & 60,1 & 17,9 & 73,9 & 11,8 & 71,2 & 17,6 & 58,8 & 11,8 & 72,7 & 10,1 \\
\hline P-valor & 0,629 & 0,086 & 0,344 & 0,002 & 0,113 \\
\hline
\end{tabular}

Status da profissão

\begin{tabular}{lcccccccccc}
\hline Ativo & 61,2 & 16,3 & 71,4 & 14 & 71,8 & 13,9 & 65,7 & 13,4 & 70 & 12,5 \\
\hline Inativo & 58,5 & 18,6 & 74,9 & 13,1 & 71,5 & 16 & 70 & 14,3 & 72,9 & 13,4 \\
\hline p-valor & 0,274 & 0,67 & 0,89 & 0,033 & 0,121 \\
\hline
\end{tabular}

Status inativo

\begin{tabular}{lcccccccccc}
\hline Aposentado por tempo & 61,4 & 16,9 & 75,7 & 10,9 & 73,7 & 14,6 & 71,3 & 13,4 & 74,3 & 13,5 \\
\hline Aposentado por invalidez & 54,3 & 18 & 73,4 & 14,2 & 69,2 & 17,4 & 68,1 & 13,9 & 70,8 & 13,5 \\
\hline Auxílio doença & 50,1 & 26,3 & 73,3 & 20,9 & 60,4 & 20,1 & 70,8 & 16,8 & 64,2 & 13,1 \\
\hline p-valor & 0,054 & 0,651 & 0,021 & 0,593 & 0,042 \\
\hline
\end{tabular}

Renda familiar 


\begin{tabular}{lcccccccccc}
\hline Menos que 1 salário & 57 & 15,5 & 67,6 & 15 & 64,7 & 19,7 & 64,2 & 12,9 & 64,7 & 14,6 \\
\hline 1 a 2 salários & 58,1 & 17,6 & 72,4 & 13,5 & 70,2 & 14,3 & 66,8 & 13 & 71,1 & 13,2 \\
\hline 3 a 5 salários & 67,5 & 15,7 & 80,1 & 10,8 & 81,2 & 12,4 & 74,9 & 14,8 & 76,4 & 10,8 \\
\hline p-valor & 0,006 & 0,001 & & 0 & & 0,001 & & 0,005 \\
\hline Quanto tempo de doença & 7 & & & & & & & \\
\hline Menos de 1 ano & 64,3 & 21,3 & 74,3 & 17,1 & 73,6 & 14,2 & 73,2 & 15,6 & 70 & 12,9 \\
\hline 1 a 3 anos & 60,8 & 18,1 & 75,2 & 13,3 & 74,8 & 14,2 & 69,1 & 13,3 & 73,3 & 12,4 \\
\hline 3 a 5 anos & 59,7 & 13,7 & 68,9 & 12,7 & 66,1 & 19,4 & 64,1 & 13,8 & 68,4 & 15,3 \\
\hline 5 anos ou mais & 54,1 & 16,5 & 70,6 & 14,5 & 68,5 & 13,9 & 64,4 & 12,9 & 70,3 & 12 \\
\hline 5 & 59,3 & 17,1 & 75,2 & 11 & 72,1 & 13,8 & 69,9 & 13,7 & 73,4 & 13,9 \\
\hline p-valor & 0,179 & 0,134 & 0,042 & 0,031 & 0,323 \\
\hline
\end{tabular}

Periodicidade/consultas

\begin{tabular}{lcccccccccc}
\hline Semanalmente & 50,3 & 19,5 & 71,5 & 11,4 & 61,1 & 17,5 & 65,1 & 14,1 & 65 & 11,7 \\
\hline Quinzenalmente & 54,6 & 18 & 68,1 & 16,3 & 65,7 & 17,6 & 62,1 & 11,8 & 64,4 & 17,6 \\
\hline Mensalmente & 59,6 & 17,4 & 72,7 & 13 & 72,1 & 15,8 & 69,1 & 13,4 & 70,7 & 13,2 \\
\hline Semestralmente & 60,8 & 17,2 & 71,8 & 14,1 & 72,8 & 10,9 & 64 & 11,9 & 72,1 & 9,9 \\
\hline Anualmente & 63,1 & 17,1 & 80,6 & 13 & 74,2 & 16,1 & 77 & 15,1 & 77,9 & 12,9 \\
\hline p-valor & 0,164 & 0,012 & 0,038 & & 0 & 0,002
\end{tabular}

$¥$ Análise de Variância One Way - Post Hoc Sheffé. DP = Desvio Padrão

Fonte: Autores (2019)

O perfil sociodemográfico dos usuários com diagnóstico de DCNT vem ao encontro de pesquisas que obtiveram resultados semelhantes ${ }^{(10-11)}$. No que tange ao perfil clínico, a literatura apresenta correlação com os resultados encontrados, os quais apontam que, dentre as DCNT mais recorrentes, encontram-se as doenças cardiovasculares, respiratórias crônicas, doenças renais crônicas, neoplasias e diabetes ${ }^{(1)}$.

A OV possui um conceito amplo que abrange a complexidade de inter-relacionarse com aspectos objetivos e subjetivos de bem-estar físico e pessoal, sendo a avaliação objetiva focada nos indicadores de saúde físicos e suas limitações e subjetiva na avaliação do indivíduo nas dimensões física, psicológica, social e ambiental ${ }^{(9)}$.

Autores pontuam que portadores de DCNT classificam sua OV como ruim, principalmente no domínio físico, evidenciando que a QV é prejudicada pelas capacidades físicas e tem repercussões importantes durante 0 tratamento ${ }^{(8)}$. Esse fato pode ser correlacionado com estudo realizado com diabéticos, uma vez que a menor média apresentada foi neste domínio, justificada pelas alterações visuais que o DM pode causar (além de lesões de pele e amputações) ${ }^{(12)}$.

Dessa forma, esses aspectos podem interferir direta ou indiretamente na QV, uma vez que autores afirmam que as circunstâncias impostas por estas doenças promovem alterações nos processos fisiológicos e influenciam em limitações na vida cotidiana, corroborando para a debilidade do estado de saúde e a dependência funcional e facilitando a simultaneidade de outras patologias ${ }^{(13)}$. 
Achados do estudo em tela evidenciam que os respondentes tem uma boa $\mathrm{QV}$, como em pesquisa realizada em Belo Horizonte-MG cujos achados revelam relação positiva da QV nos domínios Físico e Psicológico em indivíduos com idade avançada ${ }^{(14)}$. No entanto, em relação ao domínio Meio Ambiente, os resultados divergem do presente estudo, pois constatou-se que este domínio obteve pontuação baixa, o que influenciou negativamente na QV dos respondentes.

Infere-se que os resultados relacionados à diferença estatística à cor/raça prevaleceram, visto que na contemporaneidade indivíduos brancos têm mais oportunidades de atuação, acesso a informações e segurança na vida diária, quando comparados aos de cor amarela/ negra. Em contraponto, devido a um fator cultural, pessoas da cor amarela/negra são mais vulneráveis devido a determinantes sociais e em conseguinte, muitas vezes, tem mais dificuldade de ingressar no mercado de trabalho, o que influencia nos recursos financeiros com vistas a satisfazer suas necessidades, influenciando negativamente percepção da $\mathrm{QV}^{(15)}$.

Pesquisa identificou que, com o desenvolvimento das DCNT, os indivíduos passam a apresentar menores índices de produtividade, e, consequentemente, pior percepção de QV (16). Em contraponto, neste estudo, dados demostram que os usuários possuem boa QV. Entende-se que isto pode estar associado às relações pessoais que os permeiam, aceitação da doença e segurança nos cuidados de saúde e vida cotidiana.

O desenvolvimento da atividade laboral pode ser comprometido, pois mudanças influenciam negativamente o cotidiano do portador de DCNT, devido à dificuldade de conciliar trabalho com o processo de saúde doença. Estudo com portadores de doença renal crônica evidenciou que a maioria não consegue se manter no mercado de trabalho, sendo necessário receber auxílio-doença (por vezes inferior à sua renda antes do adoecimento); os problemas financeiros abalam não só o portador de DCNT, mas tem repercussões familiares e influência em outros aspectos da vida como alimentação, lazer, transporte, entre outros ${ }^{(17)}$.

Sobre o status inativo, as diferenças significativas ficaram a cargo do domínio das Relações Sociais e QV Global, em que os investigados do grupo auxílio-doença apresentaram média para QV significativamente menor, quando comparado aos casos que relataram aposentadoria por tempo de serviço. Isto pode se relacionar ao afastamento laboral precoce e, consequentemente, à diminuição do contato com pessoas/amigos, reflete na $\mathrm{OV}$, uma vez que a convivência com outras pessoas, o diálogo, expressão de emoções e o compartilhar de medos e angústias é primordial para indivíduos com situações de cronicidade ${ }^{(18)}$.

Verificou-se que a renda familiar foi a variável que mais impactou na $\mathrm{QV}$, pois diferiu significativamente em todos os domínios. Fato que vem ao encontro de estudo desenvolvido na cidade de Goiânia-GO, que revelou importante representatividade estatística no item renda familiar, demostrando que a população que tem mais condições financeiras possui melhor QV, uma vez que dispõe de mais recursos para suprir suas necessidades, o que proporciona melhor e maior satisfação quanto à saúde física e psicológica(19).

Em relação ao tempo de diagnóstico da doença, as diferenças significativas ficaram a cargo dos domínios das Relações Sociais e Meio Ambiente. No primeiro, as médias para QV dos tempos menor de um e de um a três anos foram maiores que os casos com tempo de doença de três a cinco anos. Para o domínio do Meio Ambiente, a média para QV foi significativamente maior no grupo com menos de um ano em comparação àqueles com tempos de três a cinco e cinco ou mais. Para tanto, é possível afirmar que a presença de uma DCNT causa impacto na saúde do indivíduo/população e, quanto maior o tempo de diagnóstico, mais influência a doença tem. Neste interim, quando abordadas questões referentes à $\mathrm{QV}$, é inerente que as condições sociais, ambientais e econômicas influenciam nas condições de saúde da população(20).

Quanto à periodicidade de consultas, as diferenças significativas mostraram-se 
presentes na maioria dos domínios, exceto o Físico. De modo geral, as médias para a QV no tratamento com frequência mensal foi significativamente superior quando comparada às médias das frequências semanal e quinzenal. Salienta-se que ter uma DCNT e os problemas de saúde decorrentes desta são contribuintes para o maior acesso da população aos serviços de saúde. Ressalta-se, ainda, que o uso contínuo de medicações, em especial polifarmácia e em idosos, efeitos colaterais por interações medicamentosas e agravos decorrentes das DCNT podem influenciar negativamente a QV dos usuários ${ }^{(21)}$.

A partir desse contexto, a equipe de saúde da APS tem papel importante, pois através do acolhimento ao usuário na unidade e de atividades de educação em saúde continuadas, pode disseminar conceitos e cuidados com vistas a evitar as DCNT, bem como suas complicações. Ainda é importante a atuação do enfermeiro, que cotidianamente tem sido a referência nos serviços de saúde e, através do vínculo e de sua atenção aos pacientes, pode contribuir na prevenção, bem como no cuidado com as famílias adstritas a unidade de saúde e consequentemente melhorar a $\mathrm{QV}^{(22)}$.

Outro estudo realizado com pacientes estomizados aponta que a ansiedade e depressão contribuem para maior comprometimento da QV, portanto, esses aspectos devem ser observados e identificados pelas equipes que os assistem ${ }^{(23)}$.

Neste sentido, os achados deste estudo poderão colaborar para o planejamento das ações em saúde das equipes de saúde da APS, em especial do fazer da enfermagem, com vistas a planejar o cuidado às pessoas com DCNT e ao empoderamento deles para enfrentar os desafios impostos pela doença.

Como limitação do estudo, destaca-se a coleta de dados realizada em somente um município do Sul do Brasil, o que impede que seus resultados possam ser generalizados.

\section{CONCLUSÃO}

A QV geral foi considerada satisfatória. Os domínios apresentaram os escores respectivamente psicológico, relações sociais, meio ambiente e físico $(73,3 ; 71,7 ; 68,1$; e $59,7)$. A QV apresentou relação estatística com idade, cor, status profissional, renda, tempo de doença e periodicidade das consultas.

O estudo permitiu conhecer do perfil dos pacientes com DCNT, a qualidade de vida e sua relação com as características sociodemográficas e clínicas dos pacientes assistidos na atenção primária, bem como as doenças mais incidentes no local do estudo, fato que pode direcionar e fortalecer ações de educação em saúde desenvolvidas pelas equipes de atenção primária, com vistas à qualidade de vida, em especial no domínio físico, que obteve menor escore.

\section{REFERÊNCIAS}

1. Malta DC, Bernal RTI, Lima MG, Araújo SSC de, Silva MMA da, Freitas MI de F, et al. Doenças crônicas não transmissíveis e a utilização de serviços de saúde: análise da Pesquisa Nacional de Saúde no Brasil. Rev. Saúde Pública. [Internet]. 2017 [acesso em 04 maio 2019]; 51(supl1). Disponível em: https://doi. org/10.1590/S1518-8787.2017051000090.

2. Ministério da saúde (BR). 57,4 milhões de brasileiros têm pelo menos uma doença crônica. [Internet]. Ministério da Saúde; 2014 [acesso em 04 maio 2019]. Disponível em: http://www.blog.saude.gov.br/ 
index.php/34861-57-4-milhoes-de-brasileiros-tem-pelo-menos-uma-doenca-cronica.

3. Ministério da Saúde. Portaria n. 2.436, de 21 de setembro de 2017. Aprova a Política Nacional de Atenção Básica, estabelecendo a revisão de diretrizes para a organização da Atenção Básica, no âmbito do Sistema Único de Saúde (SUS). Diário Oficial da União. 2017 set. 21; Disponível em: https://bvsms. saude.gov.br/bvs/saudelegis/gm/2017/prt2436 2209 2017.html.

4. Rodrigues MM. Martins L, Silva DMGV da, Meirelles BHS, Arruda C, Reckziegel JCL. Percepção de pessoas com doença crônica acerca da internação hospitalar. Rev. Enferm. UFPE. [Internet]. 2017 [acesso em 20 mar 2019]; 11(6). Disponível em: https://periodicos.ufpe.br/revistas/revistaenfermagem/article/ viewFile/23399/19059.

5. Tonini IG de O, Luz F do R da, Menon PD, Zanelatto C, Mazur CE. Perception of health and nutritional status of hospitalized patients with chronic diseases. ABCS Health Sci, [Internet]. 2019 [acesso em 04 maio 2019]; 44(1). Disponível em: https://doi.org/10.7322/abcshs.v44i1.1095.

6. THE Whoqol Group. The World Health Organization Quality of Life assessment (WHOQOL): position paper from the World Health Organization. Soc. Sci. Med. [Internet]. 1995 [acesso em 20 mar 2019]; 41(10). Disponível em: https://doi.org/10.1016/0277-9536(95)00112-k.

7. Cruz DSM da, Collet N, Nóbrega VM. Qualidade de vida relacionada à saúde de adolescentes com dm1- revisão integrativa. Ciênc. saúde coletiva [Internet]. 2018 [acesso em 04 maio 2019]; 23(3). Disponível em: https://doi.org/10.1590/1413-81232018233.08002016.

8. Pereira DN, Tolentino GP, Soares V, Venâncio PEM. Qualidade de vida de pessoas com doenças crônicas. Cinergis. [Internet]. 2017 [acesso em 21 out 2019]; 18(3). Disponível em: http://dx.doi. org/10.17058/cinergis.v18i3.9320.

9. Fleck MPA, Louzada S, Xavier M, Chachamovich E, Vieira G, Santos L, Pinzon V. Aplicação da versão em português do instrumento abreviado de avaliação da Qualidade de vida "Whoqolbref". Rev Saúde Pública. [Internet]. 2000 [acesso em 04 maio 2019]; 34(2). Disponível em: https://doi.org/10.1590/S003489102000000200012.

10. Sturmer J, Bettinelli LA, Amaral PP do, Bortoluzzi EC, Doring M. Perfil sociodemográfico e clínico de idosos usuários das estratégias de saúde da família. Rev enferm UFPE. [Internet]. 2017 [acesso em 21 out 2019]; 11(8). Disponível em: https://periodicos.ufpe.br/revistas/revistaenfermagem/article/ view/110189/22075.

11. Sato T de O, Fermiano NTC, Batistão MV, Moccellin AS, Driusso P, Mascarenhas SHZ. Doenças crônicas não transmissíveis em usuários de Unidades de Saúde da Família - prevalência, perfil demográfico, utilização de serviços de saúde e necessidades clínicas. R. Bras. Ciênc. Saúde. [Internet]. 2017 [acesso em 21 out 2019]; 21(1). Disponível em: https://doi.org/10.4034/RBCS.2017.21.01.05.

12. Moreschi C, Rempel C, Siqueira DFde, Backes DS, Pissaia LF, Grave MTQ. Family health strategies: profile/quality of life of people with diabetes. Rev Bras Enferm [Internet]. 2018 [acesso em 21 out 2019]; 71(6). Disponível em: https://doi.org/10.1590/0034-7167-2018-0037.

13. Zanesco C, Bordin D, Santos CB dos, Muller EV, Fadel CB. Fatores que determinam a percepção negativa da saúde de idosos brasileiros. Rev. bras. geriatr. gerontol. [Internet]. 2018 [acesso em 21 out 2019]; 21(3). Disponível em: https://doi.org/10.1590/1981-22562018021.170210.

14. Miranda LCV, Soares SM, Silva PAB. Qualidade de vida e fatores associados em idosos de um Centro de Referência à Pessoa Idosa. Ciênc saúde coletiva. [Internet]. 2016 [acesso em 21 out 2019]; 21(11). Disponível em: https://doi.org/10.1590/1413-812320152111.21352015.

15. Ministério da saúde (BR). Política Nacional de Saúde Integral da População Negra - Uma Política do SUS. 3 ed. [Internet]. Ministério da Sáude; 2017. Disponível em: https://bvsms.saude.gov.br/bvs/ publicacoes/politica nacional saude populacao negra 3d.pdf.

16. Cruz MF da, Ramires W, Wendt A, Mielke GI, Martinez-Mesa J, Wehrmeister FC. Simultaneidade de fatores de risco para doenças crônicas não transmissíveis entre idosos da zona urbana de Pelotas, Rio 
Grande do Sul, Brasil. Cad. Saúde Pública. [Internet]. 2017 [acesso em 21 out 2019]; 33(2). Disponível em: https://doi.org/10.1590/0102-311x00021916.

17. Marinho CLA, Oliveira JF de, Borges JE da S, Silva RS da, Fernandes FECV. Qualidade de vida de pessoas com doença renal crônica em hemodiálise. Rev Rene. [Internet]. 2017 [acesso em 21 out 2019]; 18(3). Disponível em: http://doi.org/10.15253/2175-6783.2017000300016.

18. Jesus NM, Souza GF de, Mendes-Rodrigues C, Almeida Neto OP de, Rodrigues DDM, Cunha CM. Qualidade de vida de indivíduos com doença renal crônica em tratamento dialítico. J. Bras. Nefrol. [Internet]. 2019 [acesso em 21 out 2019]; 41(3). Disponível em: https://doi.org/10.1590/2175-8239JBN-2018-0152.

19. Faria FL de, Labre MM, Sousa IF de, Almeida RJ de. Avaliação da qualidade de vida em pacientes com estomia intestinal. Arq. Ciênc. Saúde. [Internet]. 2018 [acesso em 21 out 2019]; 25(2). Disponível em: https://doi.org/10.17696/2318-3691.25.2.2018.924.

20. Carrapato P, Correia P, Garcia B. Determinante da saúde no Brasil: a procura da equidade na saúde. Saúde Soc. [Internet]. 2017 [acesso em 21 out 2019]; 26(3). Disponível em: https://doi.org/10.1590/S010412902017170304.

21. Becker RM, Heidemann IVSB, Meirelles BHS, Costa MFBNA da, Antonini FO, Durand MK. Práticas de cuidado dos enfermeiros a pessoas com Doenças Crônicas Não Transmissíveis. Rev bras enferm [Internet]. 2018 [acesso em 21 out 2019]; 71(supl 6). Disponível em: http://dx.doi.org/10.1590/0034-7167-20170799.

22. Santana PPC, Ramos ADV, Campos CE, Andrade M, Menezes, HF de, Camacho, ACLF, et al. O impacto da polifarmácia na qualidade de vida de idosos. Rev enferm UFPE on line. [Internet]. 2019 [acesso em 21 dez 2020]; 13(3). Disponível em: https://pesquisa.bvsalud.org/portal/resource/pt/ biblio-1015758.

23. Alievi MF, Loro MM, Franz LBB, Pluta P, Kolankiewicz ACB. Reflexos da ansiedade e depressão na qualidade de vida de pacientes estomizados. Rev. Contexto \& Saúde. [Internet]. 2020 [acesso em 21 dez 2020]; 20(41). Disponível em: https://doi.org/10.21527/2176-7114.2020.41.90-98. 


\section{QUALIDADE DE VIDA DE USUÁRIOS COM DOENÇAS CRÔNICAS NÃO TRANSMISSÍVEIS ASSISTIDOS NA ATENÇÃO PRIMÁRIA À SAÚDE}

\section{RESUMO:}

Objetivo: avaliar a Qualidade de Vida e relação com características demográficas e clínicas de usuários com Doenças Crônicas não Transmissíveis, assistidos na Atenção Primária à Saúde. Método: estudo transversal com indivíduos diagnosticados com doença crônica não transmissível, desenvolvido com questionário sociodemográfico/clínico e WHOQOL-Bref, na Atenção Primária à Saúde de um município do noroeste do Rio Grande do Sul - Brasil, entre julho de 2018 e junho 2019. Resultados: qualidade de vida com menor média no domínio Físico $(59,71)$ e maior no domínio Psicológico (73,30). Resultados com diferença estatística para faixa etária nos domínios Físico $(p<0,048)$, Psicológico $(p=0,041)$ e Meio Ambiente $(p=0,003)$, e com as variáveis cor e profissão no domínio Meio Ambiente $(p=0,002)$. Conclusão: o estudo contribui para direcionar e fortalecer ações de educação em saúde desenvolvidas pelas equipes de atenção primária, com vistas à qualidade de vida.

DESCRITORES: Doença Crônica; Atenção Primária à Saúde; Qualidade de Vida; Doenças não Transmissíveis; Enfermagem em Saúde Comunitária.

\section{CALIDAD DE VIDA DE LOS USUARIOS CON ENFERMEDADES CRÓNICAS NO TRANSMISIBLES ATENDIDOS EN ATENCIÓN PRIMARIA}

\section{RESUMEN:}

Objetivo: evaluar la Calidad de Vida y su relación con las características demográficas y clínicas de los usuarios con Enfermedades Crónicas No Transmisibles, atendidos en Atención Primaria. Método: estudio transversal con individuos diagnosticados de enfermedad crónica no transmisible, desarrollado con cuestionario sociodemográfico/clínico y WHOQOL-Bref, en la Atención Primaria de Salud de un municipio del noroeste de Rio Grande do Sul - Brasil, entre julio de 2018 y junio de 2019. Resultados: calidad de vida con menor media en el dominio Físico $(59,71)$ y mayor en el dominio Psicológico $(73,30)$. Resultados con diferencia estadística para el grupo de edad en los dominios Físico $(p<0,048)$, Psicológico $(p=0,041)$ y Ambiente $(p=0,003)$, y con las variables color y profesión en el dominio Ambiente $(p=0,002)$. Conclusión: el estudio contribuye a dirigir y reforzar las acciones de educación sanitaria desarrolladas por los equipos de atención primaria, con vistas a la calidad de vida.

DESCRIPTORES: Enfermedad crónica; Atención Primaria de Salud; Calidad de vida; Enfermedades no Transmisibles; Enfermería en Salud Comunitaria.

Recebido em: 29/07/2020

Aprovado em: 24/03/2021

Editora associada: Luciana Alcântara Nogueira

Autor Correspondente:

Adriane Cristina Bernat Kolankiewicz

Universidade Regional do Noroeste do Estado do Rio Grande do Sul - ljuí, RS, Brasil

E-mail: adri.saudecoletiva@gmail.com

\section{Contribuição dos autores:}

Contribuições substanciais para a concepção ou desenho do estudo; ou a aquisição, análise ou interpretação de dados do estudo - Pasquetti PN, Kolankiewicz ACB, Loro MM; Elaboração e revisão crítica do conteúdo intelectual do estudo - Pasquetti PN, Kolankiewicz ACB, Flôres GC, Winter VDB, Trindade LF, Bandeira LR, Loro MM; Aprovação da versão final do estudo a ser publicado - Pasquetti PN, Kolankiewicz ACB, Flôres GC, Winter VDB, Trindade LF, Bandeira LR, Loro MM; Responsável por todos os aspectos do estudo, assegurando as questões de precisão ou integridade de qualquer parte do estudo - Pasquetti PN, Kolankiewicz ACB, Flôres GC, Winter VDB, Trindade LF, Bandeira LR, Loro MM. Todos os autores aprovaram a versão final do texto.

Copyright @ 2021 Este é um artigo em acesso aberto distribuído nos termos da Licença Creative Commons Atribuição, que permite o uso irrestrito, a distribuição e reprodução em qualquer meio desde que o artigo original seja devidamente citado. 